\title{
Possibility of multi-conformational structure of mismatch DNA nucleobase in the presence of silver(I) ions
}

\author{
Hideaki Miyachi $^{\mathrm{a}, \mathrm{b}, *}$, Toru Matsui ${ }^{\mathrm{b}, \mathrm{c}}$, Yasuteru Shigeta $^{\mathrm{d}}$, Koichi Yamashita $^{\mathrm{e}}$, Kimihiko Hirao $^{\mathrm{b}, \mathrm{f}, *}$ \\ ${ }^{a}$ Department of Medicine, School of Medicine, Chiba University, 1-8-1 Inohana, Chuo-ku, Chiba-shi, Chiba 260-8670, \\ Japan \\ ${ }^{b}$ Department of Applied Chemistry, School of Engineering, The University of Tokyo, 7-3-1 Hongo, Bunkyo-ku, Tokyo \\ 113-8656, Japan \\ ${ }^{c}$ Department of Chemistry, Graduate School of Science, Osaka University, 1-1 Machikaneyama, Toyonaka, Osaka \\ 560-0043, Japan \\ ${ }^{d}$ Picobiology Institute, Graduate School of Life Science, University of Hyogo, 3-2-1 Kouto, Kamigouri-cho, Ako-gun, \\ Hyogo 678-1297, Japan \\ ${ }^{e}$ Department of Chemical Engineering, School of Engineering, The University of Tokyo, 7-3-1 Hongo, Bunkyo-ku, \\ Tokyo 113-8656, Japan \\ ${ }^{f}$ Advanced Science Institute, RIKEN, 2-1 Hirosawa, Wako, Saitama 351-0198, Japan
}

\begin{abstract}
Geometrical and electronic structures of uracil nucleobase pair containing $\mathrm{Ag}^{\mathrm{I}}$ ions have been studied theoretically and computationally. Four stable conformers are expected to co-exist under normal experimental conditions, and transition state structures among the conformers are also presented. The replacement of protons with $\mathrm{Ag}^{\mathrm{I}}$ ions increases the size of the mismatch nucleobase pairs and leads to a better match of the size stabilizing the duplex. From the calculated infrared, Raman and UV absorption spectra of each conformer and mixtures of all conformers, all the conformers are expected to make significant contributions to the experimental spectroscopic profiles.
\end{abstract}

\section{Introduction}

Deoxyribonucleic acid (DNA) contains genetic instructions used in the development and functioning of living organisms. Chemists' interest in DNA has recently been focused on its flexible functioning of living organisms. One of the promising approaches to modify its properties is to coordinate metal ions to nucleobases, neucleosides or nucleotides to form metal-complexed DNA.

The nucleobase moiety of DNA interacts with metal ions in various ways. For example, $\mathrm{Pt}^{\mathrm{II}}$ complexes, such as cisplatin known as an antitumor drug, distorts the structure of DNA duplex by coordinating to the guanine N7 atom located in the outer region of the duplex [1-5]. There are reports that metal ions such as $\mathrm{Ni}^{\mathrm{II}}, \mathrm{Zn}^{\mathrm{II}}$ and $\mathrm{Mg}^{\mathrm{II}}$ ions also interact with DNA in the same way [6-9]. On the other hand, metal ions can be captured in between the nucleobase pairs as well forming metal-nucleobase bonds in place of the hydrogen bonds which originally exist in

${ }^{*}$ Corresponding authors. FAX: +81358418745

Email addresses: z7m1101@students. chiba-u.jp (Hideaki Miyachi), hirao@riken.jp (Kimihiko Hirao)

Preprint submitted to Chemical Physics Letters

June 9, 2010

(C) 2010. This manuscript version is made available under the Elsevier user license

http://www.elsevier.com/open-access/userlicense/1.0/ 
between the nucleobases [10-25]. Ono et al. demonstrated that a thymine-thymine (T-T) [16$18,25]$ and cytocine-cytocine [24] mismatch nucleobase pairs could selectively capture $\mathrm{Hg}^{\mathrm{II}}$ and $\mathrm{Ag}^{\mathrm{I}}$ ions forming metal-mediated nulceobase pairs in duplexes. Since the binding of $\mathrm{Hg}^{\mathrm{II}}$ and $\mathbf{T}-\mathbf{T}$ is highly selective and results in thymine- $\mathrm{Hg}^{\mathrm{II}}$-thymine $\left(\mathbf{T}-\mathrm{Hg}^{\mathrm{II}}-\mathbf{T}\right)$, a DNA-based sensor capable of selectively detecting $\mathrm{Hg}^{\mathrm{II}}$ ions in aqueous solutions containing various heavy metal ions have been developed [26]. This is a typical use of DNA nucleobases for new materials. Thus, a method for capturing desired metal ions in DNA strands is a potential source for new materials such as sensors or DNA-based wire with electronic conductivity [27, 28].

Theoretical and computational chemistry based on quantum mechanics are powerful methods to explore effects of metal complexation from microscopic and electronic points of view $[3,4$, 19-23, 29]. Following the work by Ono et al., our recent theoretical and computational study has elucidated the structural properties, electronic structures and UV absorption spectra of T-T and $\mathbf{T}-\mathrm{Hg}^{\mathrm{II}}-\mathbf{T}$ [19]. We have also confirmed by calculation of interaction energy [30-32] that a thymine- $\mathrm{Ag}^{\mathrm{I}}$-thymine $\left(\mathbf{T}-\mathrm{Ag}^{\mathrm{I}}-\mathbf{T}\right)$ cannot exist in a duplex without significant structural distorsion due to an overall negative charge.

Ono et al. have also suggested (in Scheme 2 of Ref. [25]) that more than one $\operatorname{Ag}^{\mathrm{I}}$ ion may be bound between two 5-substituted-1-methyl-uracil (N) in a DNA duplex. However, the exact coordination geometry of the system has not yet been clarified, since no reliable X-ray crystal data are available at this point. Therefore, in the present letter we report our theoretical and computational evidence on the coordination geometry of uracil pair containing multiple $\mathrm{Ag}^{\mathrm{I}}$ ions, which supports the suggestion of Ono's group. Based on our calculations, we propose that several conformers co-exist in their experimental conditions. Furthermore, we present calculated spectroscopic data, which will hopefully contribute to experimental studies, by taking estimated properties of these conformers into account.

We focus our present study on a single 1-methyl-uracil (U) pair (U-U). As mentioned above, previous studies indicated that $\mathbf{U}-\mathbf{U}$ containing one $\mathrm{Ag}^{\mathrm{I}}$ silver ion cannot stack in a duplex without substantial structural distorsion due to strong electrostatic repulsion [19]. In addition, a profile plot of the thermally induced transition temperature versus the $\mathrm{Ag}^{\mathrm{I}}$ ion concentration suggests that two $\mathrm{Ag}^{\mathrm{I}}$ ions are bound to $\mathbf{U}-\mathbf{U}$ [25]. Thus, $\mathbf{U}-\mathbf{U}$ containing two $\mathrm{Ag}^{\mathrm{I}}$ ions $\left(\mathbf{U}-\mathrm{Ag}_{2}-\mathbf{U}\right)$ has been calculated in our study. The backbone atoms of the duplex have been substituted by the methyl group in order to perform first principle quantum mechanical calculations for $\mathbf{U}, \mathbf{U}-\mathbf{U}$ and $\mathbf{U}-\mathrm{Ag}_{2}-\mathbf{U}$.

Since the major aim of our study is to elucidate the structure and the physical properties such as IR, Raman and UV absorption spectra of $\mathbf{N}-\mathrm{Ag}_{2}-\mathbf{N}$, the effect of backbone atoms and 5-substituents has been left out for future studies. The cis-type $\mathbf{U}-\mathbf{U}$ and $\mathbf{U}-\mathrm{Ag}_{2}-\mathbf{U}$, where the N1 nitrogen atoms of respective $\mathbf{U}$ are placed on the same side, have been investigated based on the cis-type $\mathbf{U}-\mathbf{U}$ found in the X-ray diffraction data to $2.0 \AA$ resolution of the polymerase active site [19] (PDB ID: 1NJY [33]).

\section{Computational detail}

Each stable and transition state structure described in this article was geometry-optimized by density functional theory (DFT) calculations with B3LYP hybrid functional [34-38]. For the calculations of excitation energies and associated oscillator strengths, time dependent-DFT (TDDFT) calculations were performed using B3LYP hybrid functional (TD-B3LYP). Since we are interested in the excitations with large absorbance, we only calculated singlet-singlet excitations. 
The effect of bulk solvent was taken into account by the polarized continuum model (PCM) [3941] using the radii proposed by Pauling and with water as solvent. The basis sets used to carry out our calculations were Aug-cc-pVTZ with relativistic pseudopotential (Aug-cc-pVTZ+pp) for $\mathrm{Ag}$ atoms $[42,43]$ and $6-31++\mathrm{G}(\mathrm{d}, \mathrm{p})[44,45]$ for $\mathrm{C}, \mathrm{H}, \mathrm{N}$ and $\mathrm{O}$ atoms. The natural bond orbital (NBO) analysis $[46,47]$ was used for the population analysis of charges and bond orders. All the calculations were performed using the Gaussian 03 program package $[48,49]$. The convergence criterion of SCF was set to "Tight", and the "UltraFine" grid was used for numerical integration in DFT calculations as implemented in the Gaussian 03.

\section{Results and discussion}

\subsection{Structures and relative energies of conformers}

Since each of the two $\mathrm{Ag}^{\mathrm{I}}$ ions can mediate a bond in a duplex by binding to N3, O2 and O4 atoms, we calculated all four possible stable conformers shown in Fig. 1, denoted as A, $\mathbf{B}, \mathbf{C}$ and $\mathbf{D}$. In addition, transition state structures from $\mathbf{A}$ to $\mathbf{B}$ and $\mathbf{C}$ (TS_AB and TS_AC, respectively) were also calculated. Selected key bond lengths, angles and dihedral angles are summarized in Table 1. In all six structures, the N3a-Ag1-Ag2-N3b and N3a-N1a-N1b-N3b dihedral angles in the table show that the two $\mathbf{U}$ and $\mathrm{Ag}^{\mathrm{I}}$ ions result in planar structures. A planar structure is also assumed to exist in the stacking nucleobase pairs in a duplex, because there are stacking interactions in addition to the strong metal-nucleobase bonds in $\mathbf{U}-\mathrm{Ag}_{2}-\mathbf{U}$, from other nucleobases in the duplex, which stabilize the planar structure. The O-Ag-N, N-Ag-N and O-Ag$\mathrm{O}$ angles were all within $170 \pm 5^{\circ}$ for stable structures, showing that the bonds mediated by the $\mathbf{A g}^{\mathrm{I}}$ ions are nearly linear. In the transition state structures, TS_AB and TS_AC, the optimized structures have one $\mathrm{Ag}^{\mathrm{I}}$ ion in the conformation of $\mathbf{A}$ moved to conformations of $\mathbf{B}$ and $\mathbf{C}$. This resulted in a change of one of the bond angles to $136 \pm 2^{\circ}$ while the other remained nearly unchanged. The Intrinsic Reaction Coordinate (IRC) calculation from TS_AB or TS_AC result in structures $\mathbf{A}$ and $\mathbf{B}$ or $\mathbf{C}$, respectively. Therefore, the calculations indicate that the conversions between $\mathbf{A}$ and $\mathbf{B}$ or $\mathbf{C}$ are one-step reactions. As shown in the figure, the displacement vectors corresponding to the imaginary frequencies of the transition structures are composed of a twist of two nucleobase pairs in a plane along with the exchange of nucleobase atoms that are bound to $\mathrm{Ag}^{\mathrm{I}}$ ions.

The energies of the six structures calculated for $\mathbf{U}-\mathrm{Ag}_{2}-\mathbf{U}$ relative to $\mathbf{A}$ and estimations of the formation energies for the capturing of $\mathrm{Ag}^{\mathrm{I}}$ ions by $\mathbf{U}-\mathbf{U}$ forming $\mathbf{U}-\mathrm{Ag}_{2}-\mathbf{U}$ are listed in Table 2 . The formation energy is defined as

$$
\Delta E_{\text {Formation }}=\left\{E\left(\mathbf{U}-\mathrm{Ag}_{2}-\mathbf{U}\right)+2 E_{\mathrm{p}}\right\}-\left\{E(\mathbf{U}-\mathbf{U})+2 E\left(\mathrm{Ag}^{\mathrm{I}}\right)\right\},
$$

where $E$ denotes the energy calculated for the corresponding entity. Since the formation of $\mathbf{U}$ $\mathrm{Ag}_{2}-\mathbf{U}$ takes place in aqueous solution, we estimated the energy of a proton in aqueous condition, $E_{\mathrm{p}}$, as the difference between $\mathrm{H}_{3} \mathrm{O}^{+}$and $\mathrm{H}_{2} \mathrm{O}, E_{\mathrm{p}}=E\left(\mathrm{H}_{3} \mathrm{O}^{+}\right)-E\left(\mathrm{H}_{2} \mathrm{O}\right)$.

The formation energies indicate that the formation of $\mathbf{A}$ and $\mathbf{B}$ are energetically favorable. Depending on conditions, $\mathbf{C}$ may also be formed while the formation of $\mathbf{D}$ is unlikely. Among the four stable conformers, $\mathbf{B}$ is $0.72 \mathrm{kcal} / \mathrm{mol}$ more stable than $\mathbf{A}$, which is the second most stable conformer. In addition, $\mathbf{C}$ is only $3.16 \mathrm{kcal} / \mathrm{mol}$ less stable than $\mathbf{B}$. Since the barrier height of the transition structures TS_AB and TS_AC are less than $7.78 \mathrm{kcal} / \mathrm{mol}$, the results indicate that $\mathbf{A}$, $\mathbf{B}$ and $\mathbf{C}$ should co-exist at room temperature or higher. The conformer $\mathbf{D}$ is less stable by more 
than $10 \mathrm{kcal} / \mathrm{mol}$ than $\mathbf{A}$. Thus, $\mathbf{D}$ should have no significant effect in the properties measured in any experiments.

One would realize that $\mathbf{U}-\mathbf{U}$ also has four possible stable structures which correspond to those of $\mathbf{U}-\mathrm{Ag}_{2}-\mathbf{U}$, since each $\mathbf{U}$ may take either amide form or imide form. For example, the $\mathbf{U}-\mathbf{U}$ in Fig. 1 (U-U(A)) corresponds to $\mathbf{A}$. The relative energy of other three conformers are higher than $\mathbf{U}-\mathbf{U}(\mathrm{A})$ by at least $9.56 \mathrm{kcal} / \mathrm{mol}$. The $\mathbf{U}-\mathbf{U}(\mathrm{A})$ should take a major role in the measured properties of $\mathbf{U}-\mathbf{U}$ due to the relative energy and the higher activation energy expected for the shift between the conformers. Therefore, the presence of the $\mathrm{Ag}^{\mathrm{I}}$ ions enables the nucleobase pair in $\mathbf{U}-\mathrm{Ag}_{2}-\mathbf{U}$ to form conformers different from $\mathbf{U}-\mathbf{U}$.

\subsection{Size match of the nucleobase pair in a duplex}

In addition to the interaction between the nucleobase pairs, the stability of a nucleobase pair in a duplex also depends on the size match of the nucleobase pair to a space between stiff phosphate-deoxyribose backbones. In the experiment by Ono et al., the $\mathbf{N}-\mathbf{N}$ is stacked in between two adenine-thymine pairs (A-T) (Scheme 1 in Ref. [25]). To estimate the distance between the nucleobases, we have selected the following fourteen X-ray diffraction data of the polymerase active sites containing short DNA duplexes with duplex lengths similar to the duplex studied in the experiment by Ono et al.: PDB ID: 1NJW, 1NJX, 1NJY, 1NJZ, 1NK0, 1NK4, $1 \mathrm{NK} 5,1 \mathrm{NK} 6,1 \mathrm{NK} 7,1 \mathrm{NK} 8,1 \mathrm{NK} 9,1 \mathrm{NKB}, 1 \mathrm{NKC}$ and 1NKE [33].

The average distance between the N9 of adenine and N1 of T averaged over $56 \mathbf{A}-\mathbf{T}$ is $8.88 \pm 0.70 \AA$ while our calculated value is $9.11 \AA$. The N1-N1 distance of T-T found in one of the X-ray diffraction data (PDB ID: $1 \mathrm{NJY}$ ) is $7.38 \AA$, while the calculated value for $\mathbf{U}$-U is 7.36 $\AA$ A, both being significantly shorter than the distance between nucleobases in A-T listed above. The close agreement of the calculated and experimental distances suggests that the essential features of the interaction between the nucleobase pairs are captured, although our calcualtions have been carried out with a simple model. The N1a-N1b distances in all the conformers of $\mathbf{U}-\mathrm{Ag}_{2}-\mathbf{U}$ are $8.30 \AA$ or longer. The calculated values indicate that the replacement of protons with the $\mathrm{Ag}^{\mathrm{I}}$ ions increases the size of the mismatch nucleobase pairs and leads to a better match of the size in the duplex.

\subsection{Electronic structures and molecular spectra}

The results of the NBO analysis are listed in Table 3 in order to examine the electronic structure of the system. The natural charges and Wiberg bond indices of the N1, N3, O2 and O4 atoms hardly depend on the formation of $\mathbf{U}-\mathbf{U}$, showing that it is mediated by hydrogen bonds. In our previous study of the interaction of cisplatin and guanine-cytosine pair (G-C), we showed that the binding of cisplatin induces transfer of proton from cytosine to guanine resulting in a positively charged guanine and negatively charged cytosine $\left(\mathbf{G}^{*} \mathbf{C}\right)$ [3]. This charge redistribution between the two nucleobases slightly stabilizes $\mathbf{G}^{*}-\mathbf{C}$ more than $\mathbf{G}-\mathbf{C}$ by $0.72 \mathrm{kcal} / \mathrm{mol}$, which are expected to co-exist in solution in similar way to $\mathbf{U}-\mathrm{Ag}_{2}-\mathbf{U}$. As for $\mathbf{U}-\mathrm{Ag}_{2}-\mathbf{U}$, the charge redistribution induced by the binding of $\mathrm{Ag}$ ions occurs within the $\mathrm{Ag}^{\mathrm{I}}$ and nucleobases. There are significant increases in the negative charge on the $\mathrm{N} 3, \mathrm{O} 2$ and $\mathrm{O} 4$ atoms of $\mathbf{U}$, which means that there are decreases in the positive charges on $\mathrm{Ag}^{\mathrm{I}}$. Therefore, the ionic bonding properties of metal-nucleobases are enhanced. In addition, the decrease in the repulsion between two positively charged $\mathrm{Ag}^{\mathrm{I}}, E_{\text {rep }}=\left(Q_{1} Q_{2}\right) / r_{12}$, where $Q_{i}(i=1,2)$ is the charge on each atom and $r_{12}$ is the distance between the two atoms, affects the overall stability of the conformers. Precisely, the $E_{\text {rep }}$ in $\mathbf{B}$ is noticeably smaller than that of $\mathbf{A}$ and $\mathbf{C}$. Although the $E_{\text {rep }}$ between two $\mathrm{Ag}^{\mathrm{I}}$ of $\mathbf{A}$ 
and $\mathbf{C}$ are similar, $\mathbf{C}$ is unstable due to the effect of the repulsion between two $\mathrm{O} 4$. In $\mathbf{D}$, the repulsion between two $\mathrm{Ag}^{\mathrm{I}}$ hardly exists. However, the Wiberg bond indices of $\mathrm{Ag}-\mathrm{O} 2$ and $\mathrm{Ag}-\mathrm{O} 4$ are considerably smaller than that of other conformers. This suggests that the orbital relaxation caused by the interaction of $\mathrm{Ag}^{\mathrm{I}}$ ions and nucleobases is smaller and leads to the higher energy than other conformers.

Molecular spectra (UV, IR and Raman spectra) are powerful experimental tools for estimating the coordination geometry of the systems of the present concern. The UV absorption spectra of $\mathbf{U}-\mathbf{U}$ and four conformers of $\mathbf{U}-\mathrm{Ag}_{2}-\mathbf{U}$ calculated by TD-DFT are shown in Fig. 2, and two peaks for each structure are summarized in Table 4. Each of the four conformers exhibits two distinct peaks with different positions and relative oscillator strengths, which may be used to identify the coordination geometry of $\mathrm{Ag}^{\mathrm{I}}$ ions. In comparison with the spectrum of $\mathbf{U}-\mathbf{U}$, the peak at higher wavelength is relatively weakened, while the peak at lower wavelength is enhanced.

\subsection{Contributions of multiple conformers to spectroscopic profiles}

For an application to a population analysis of experimental spectra, relative populations of the four possible conformers predicted in our calculations in an aqueous solution at temperature $T$ were estimated assuming the Boltzmann distribution,

$$
P(E) \propto \exp \left(-\frac{\Delta E}{k_{\mathrm{B}} T}\right)
$$

where $\Delta E$ is the relative energy, $k \mathrm{~B}$ is the Boltzmann constant and $T$ is the temperature. The relative population of $\mathbf{B}$ is found to exceed $75 \%$ at $T=300 \mathrm{~K}$. The estimated UV spectra taking the contributions of multiple conformers into account are also plotted with the spectra of $\mathbf{B}$ in Fig. 2 (b). As seen in the figure, the theoretical estimation is almost identical with the UV absorption spectrum of $\mathbf{B}$. Nonetheless, small shifts in peak positions caused by contributions from other conformers can be observed for $\mathbf{U}-\mathrm{Ag}_{2}-\mathbf{U}$. This is basically analogous for the IR and Raman spectra (Fig. 3), but the peak intensities of the vibrational spectra are distinctly different for several peaks when the contributions of all the conformers are taken into account.

\section{Summary}

We have explored by first-principles quantum chemical calculations the possible coordination geometries of uracil nucleobase pair, $\mathbf{U}-\mathbf{U}$, in the presence of $\mathrm{Ag}^{\mathrm{I}}$ ions. The transition state structures suggest that conversions among conformers are one-step reactions. While the relative populations of four conformers of $\mathbf{U}-\mathrm{Ag}_{2}-\mathbf{U}$ estimated assuming the Boltzmann distribution predict the dominance of $\mathbf{B}$ in $\mathbf{U}-\mathrm{Ag}_{2}-\mathbf{U}$, contributions of other conformers to spectroscopic profiles are found to be significant. Despite the use of a limited model in our study due to limited computational resources, the present outcome will be used as a guideline for experimental analyses of the spectroscopic and dynamic properties of 5-substituted or other nucleobase pairs in a duplex with backbone atoms.

\section{Acknowledgement}

H. M. and T. M. are grateful to the Research Fellowships for Young Scientists from the Japan Society for the Promotion of Science (JSPS). H. M. thanks the Chiba Prefecture, Japan for the financial support. This research is supported in part by the Core Research for Evolutional 
Science and Technology (CREST) Program "High Performance Computing for Multi-Scale and Multi-Physics Phenomena" from the Japan Science and Technology Agency (JST). H. M. and T. M. are supported by Grant-in-Aid for JSPS Fellows from the Ministry of Education, Culture, Sports, Science and Technology (MEXT) of Japan. This research is also partly supported by a Grant-in-Aid for Young Scientists (B) (No. 20750004) from the JSPS.

\section{References}

[1] I.L. Zilberberg, V.I. Avdeev, G.M. Zhidomirov, J. Mol. Struct. (THEOCHEM) 418 (1997) 73.

[2] N.S. Hush, J. Schamberger, G.B. Bacskay, Coord. Chem. Rev. 249 (2005) 299.

[3] T. Matsui, Y. Shigeta, K. Hirao, Chem. Phys. Lett. 423 (2006) 331.

[4] T. Matsui, Y. Shigeta, K. Hirao, J. Phys. Chem. B 111 (2007) 1176

[5] T. Zimmermann, Z. Chval, J.V. Burda, J. Phys. Chem. B 113 (2009) 3139.

[6] N.G. Abrescia, L. Malinina, L.G. Fernandez, T. Huynh-Dinh, S. Neidle, J.A. Subirana, Nucleic Acids Res. 27 (1999) 1593.

[7] B.I. Kankia, Nucleic Acids Res. 28 (2000) 911.

[8] K.J. Baeyens, H. L. De Bondt, A. Pardi, S. R. Holbrook, Proc. Natl. Acad. Sci. U. S. A. 93 (1996) 12851.

[9] H. Robinson, Y.G. Gao, R. Sanishvili, A. Joachimiak, A.H. Wang, Nucleic Acids Res. 28 (2000) 1760.

[10] J.S. Lee, L.J. Latimer, R.S. Reid, Biochem. Cell Biol. 71 (1993) 162.

[11] M. Fuentes-Cabrera, B.G. Sumpter, J.E. Sponer, J. Sponer, L. Petit, J. C. Wells, J. Phys. Chem. B 111 (2007) 870.

[12] K. Tanaka, G. H. Clever, Y. Takezawa, Y. Yamada, C. Kaul, M. Shionoya, T. Carell, Nat. Nanotechnol. 1 (2006) 190.

[13] G.H. Clever, T. Carell, Angew. Chem. Int. Ed. 46 (2007) 250.

[14] G.H. Clever, C. Kaul, T. Carell, Angew. Chem. Int. Ed. 46 (2007) 6226.

[15] S.S. Alexandre, J.M. Soler, L. Seijo, F. Zamora, Phys. Rev. B 73 (2006) 205112.

[16] Y. Miyake, H. Togashi, M. Tashiro, H. Yamaguchi, S. Oda, M. Kudo, Y. Tanak, Y. Kondo, R. Sawa, T. Fujimoto, T. Machinami, A. Ono, J. Am. Chem. Soc. 128 (2006) 2172.

[17] Y. Tanaka, S. Oda, H. Yamaguchi, Y. Kondo, C. Kojima, A. Ono, J. Am. Chem. Soc. 129 (2007) 244.

[18] Y. Tanaka, A. Ono, Dalton Transactions 37 (2008) 4965.

[19] H. Miyachi, T. Matsui, Y. Shigeta, K. Hirao, Phys. Chem. Chem. Phys. 12 (2010) 909.

[20] T. Matsui, H. Miyachi, T. Sato, Y. Shigeta, K. Hirao, J. Phys. Chem. B 112 (2008) 16960.

[21] T. Matsui, H. Miyachi, Y. Nakanishi, Y. Shigeta, T. Sato, Y. Kitagawa, M. Okumura, K. Hirao, J. Phys. Chem. B 113 (2009) 12790.

[22] Y. Nakanishi, Y. Kitagawa, Y. Shigeta, T. Saito, T. Matsui, H. Miyachi, T. Kawakami, M. Okumura, K. Yamaguchi, Polyhedron 28 (2009) 1714.

[23] Y. Nakanishi, Y. Kitagawa, Y. Shigeta, T. Saito, T. Matsui, H. Miyachi, T. Kawakami, M. Okumura, K. Yamaguchi, Polyhedron 28 (2009) 1945.

[24] A. Ono, S. Cao, H. Togashi, M. Tashiro, T. Fujimoto, T. Machinami, S. Oda, Y. Miyake, I. Okamoto, Y. Tanaka, Chem. Commun. 39 (2008) 4825.

[25] I. Okamoto, K. Iwamoto, Y. Watanabe, Y. Miyake, A. Ono, Angew. Chem. Int. Ed. 48 (2009) 1648.

[26] A. Ono, H. Togashi, Angew. Chem. Int. Ed. 43 (2004) 4300.

[27] T. Tsukamoto, Y. Ishikawa, Y. Sengoku, N. Kurita, Chem. Phys. Lett. 474 (2009) 362.

[28] S.S. Mallajosyula, S.K. Pati, J. Phys. Chem. Lett. 1 (2010) 1881.

[29] H. Miyachi, Y. Shigeta, K. Hirao, J. Phys. Chem. A 109 (2005) 8800

[30] M. Kamiya, T. Tsuneda, K. Hirao, J. Chem. Phys. 117 (2002) 6010.

[31] T. Sato, T. Tsuneda, K. Hirao, J. Chem. Phys. 123 (2005) 104307.

[32] T. Sato, T. Tsuneda, K. Hirao, J. Chem. Phys. 126 (2007) 234114.

[33] S. J. Johnson, L. S. Beese, Cell 116 (2004) 803.

[34] A.D. Becke, Phys. Rev. A 38 (1988) 3098.

[35] A.D. Becke, J. Chem. Phys. 98 (1993) 5648.

[36] C. Lee, W. Yang, R.G. Parr, Phys. Rev. B 37 (1988) 785.

[37] B. Miehlich, A. Savin, H. Stoll, H. Preuss, Chem. Phys. Lett. 157 (1989) 200.

[38] S.H. Vosko, L. Wilk, M. Nusair, Can. J. Phys. 58 (1980) 1200.

[39] S. Miertus, E. Scrocco, J. Tomasi, Chem. Phys. 55 (1981) 117.

[40] J. Tomasi, B. Mennucci, R. Cammi, Chem. Rev. 105 (2005) 2999.

[41] M. Cossi, V. Barone, J. Chem. Phys. 115 (2001) 4708.

[42] K.A. Peterson, C. Puzzarini, Theor. Chem. Acc. 114 (2005) 283. 
[43] D. Figgen, G. Rauhut, M. Dolg, H. Stoll, Chem. Phys. 311 (2005) 227.

[44] W.J. Hehre, R. Ditchfield, J.A. Pople, J. Chem. Phys. 56 (1972) 2257.

[45] T. Clark, J. Chandrasekhar, P.v.R. Schleyer, J. Comput. Chem. 4 (1983) 294.

[46] J.P. Foster, F. Weinhold, J. Am. Chem. Soc. 102 (1980) 7211.

[47] A.E. Reed, R.B. Weinstock, F. Weinhold, J. Chem. Phys. 83 (1985) 735.

[48] M.J. Frisch et al., Gaussian 03 (Revision D.01), Gaussian, Inc., Wallingford, CT, 2004

[49] E.D. Glendening, A.E. Reed, J.E. Carpenter, F. Weinhold, NBO Version 3.1. 
Graphical Abstract, Tables and Figures Part for the manuscript by H. Miyachi et al.

Table 1: Selected key bond lengths (in $\AA$ ), angles and dihedral angles (in degrees) of $\mathbf{U}-\mathrm{Ag}_{2}-\mathbf{U}$.

\begin{tabular}{ccccccc}
\hline \hline & A & B & C & D & TS_AB & TS_AC \\
\hline Ag1-Ag2 & 2.78 & 2.77 & 2.79 & 4.32 & 2.94 & 2.95 \\
N1a-N1b & 8.67 & 8.43 & 8.30 & 8.32 & 8.51 & 8.40 \\
N3a-N3b & 4.91 & 4.28 & 4.28 & 4.24 & 4.50 & 4.47 \\
N3b-Ag1 & & & 2.15 & & & \\
N3b-Ag1 & 2.14 & & 2.15 & & 2.59 & 2.17 \\
O2a-Ag1 & & & & 2.15 & & \\
O2b-Ag1 & & & & 2.15 & & \\
O4a-Ag1 & 2.15 & 2.16 & & & 2.17 & 2.31 \\
O4b-Ag1 & & 2.16 & & & & \\
N3a-Ag2 & 2.14 & 2.15 & & & 2.16 & 2.67 \\
N3b-Ag2 & & 2.15 & & & & \\
O2a-Ag2 & & & 2.17 & & & \\
O2b-Ag2 & & & 2.17 & & & \\
O4a-Ag2 & & & & 2.16 & & \\
O4b-Ag2 & 2.16 & & & 2.16 & 2.29 & 2.17 \\
N3a-Ag1-N3b & & & 168 & & & \\
O2a-Ag1-O2b & & & & 173 & & \\
O4a-Ag1-N3b & 168 & & & & 137 & 165 \\
O4a-Ag1-O4b & & 168 & & & & \\
N3a-Ag2-N3b & & 168 & & & 168 & 135 \\
N3a-Ag2-O2b & 167 & & & & & \\
O2a-Ag2-O2b & & & 167 & & & \\
O4a-Ag2-O4b & & & & 174 & & \\
N3a-Ag1-Ag2-N3b & 180 & 180 & 180 & 180 & 180 & 180 \\
N3a-N1a-N1b-N3b & 179 & 180 & 179 & 180 & 180 & 180 \\
imaginary frequency & & & & & $64.36 i$ & $64.87 i$ \\
\hline \hline
\end{tabular}


Table 2: Relative energies and formation energies (in $\mathrm{kcal} / \mathrm{mol}$ ) of the stable and transition state structures of $\mathbf{U}-\mathrm{Ag}_{2}-\mathbf{U}$.

\begin{tabular}{ccccc}
\hline \hline & $\Delta E$ & $\Delta E_{\mathrm{ZPE}}{ }^{\mathrm{a}}$ & $\Delta G^{\mathrm{b}}$ & $\Delta E_{\text {Formation }}{ }^{\mathrm{c}}$ \\
\hline $\mathbf{A}$ & 0.00 & 0.00 & 0.00 & -0.44 \\
TS_AB & 6.23 & 5.83 & 5.89 & \\
$\mathbf{B}$ & -0.72 & -0.72 & -0.43 & -1.15 \\
TS_AC & 7.78 & 7.35 & 7.29 & \\
$\mathbf{C}$ & 2.44 & 2.40 & 2.57 & 2.00 \\
$\mathbf{D}$ & 13.41 & 13.36 & 13.17 & 12.98 \\
\hline \hline
\end{tabular}

${ }^{a}$ Relative energies including zero point energy (ZPE) correction.

b Relative Gibbs Free Energy at $T=298.15 \mathrm{~K}$.

${ }^{c}$ Negative number represents a conformer of $\mathbf{U}-\mathrm{Ag}_{2}-\mathbf{U}$ which is more stable than $\mathbf{U}-\mathbf{U}$.

Table 3: Natural charges and Wiberg bond indices in Natural Atomic Orbital basis calculated by NBO analysis for $\mathbf{U}$, $\mathbf{U}-\mathbf{U}$ and $\mathbf{U}-\mathrm{Ag}_{2}-\mathbf{U}$.

\begin{tabular}{ccccccc}
\hline \hline Natural charge & $\mathbf{U}$ & $\mathbf{U}-\mathbf{U}$ & $\mathbf{A}$ & $\mathbf{B}$ & $\mathbf{C}$ & $\mathbf{D}$ \\
\hline Ag1 & & & 0.73 & 0.79 & 0.66 & 0.75 \\
Ag2 & & & 0.74 & 0.67 & 0.82 & 0.78 \\
N1 & -0.44 & -0.43 & -0.43 & -0.43 & -0.43 & -0.42 \\
N3 & -0.64 & -0.65 & -0.73 & -0.72 & -0.73 & -0.68 \\
O2 & -0.76 & $-0.75,-0.76^{\mathrm{b}}$ & $-0.81,-0.79^{\mathrm{b}}$ & -0.79 & -0.82 & -0.82 \\
O4 & -0.77 & $-0.77,-0.76^{\mathrm{b}}$ & $-0.80,-0.79^{\mathrm{b}}$ & -0.82 & -0.80 & -0.82
\end{tabular}

\begin{tabular}{ccccccc} 
Wiberg bond indices & $\mathbf{U}$ & $\mathbf{U}-\mathbf{U}$ & $\mathbf{A}$ & $\mathbf{B}$ & $\mathbf{C}$ & $\mathbf{D}$ \\
\hline $\mathrm{N}^{\mathrm{a}}$ & 3.45 & 3.45 & 3.46 & 3.46 & 3.46 & 3.47 \\
$\mathrm{~N}^{\mathrm{a}}$ & 3.27 & 3.27 & 3.08 & 3.09 & 3.09 & 3.01 \\
$\mathrm{O}^{\mathrm{a}}$ & 1.83 & $1.85,1.83^{\mathrm{b}}$ & $1.81,1.79^{\mathrm{b}}$ & 1.80 & 1.79 & 1.78 \\
O4 $^{\mathrm{a}}$ & 1.82 & $1.81,1.85^{\mathrm{b}}$ & $1.77,1.81^{\mathrm{b}}$ & 1.80 & 1.78 & 1.78 \\
$\mathrm{Ag} 1-\mathrm{Ag} 2$ & & & 0.07 & 0.07 & 0.07 & 0.00 \\
$\mathrm{Ag} 1-\mathrm{O} 4$ & & & $0.18,0.16^{\mathrm{b}}$ & 0.17 & 0.25 & 0.19 \\
$\mathrm{Ag} 1-\mathrm{N} 3$ & & & $0.26,0.25^{\mathrm{b}}$ & 0.25 & 0.15 & 0.17 \\
\hline \hline
\end{tabular}

a Total of Wiberg bond indices for the atom.

b Two numbers are listed when the results for two nuclebases are different.

Table 4: Peak positions and intensities of UV absorption spectra calculated for $\mathbf{U}-\mathrm{Ag}_{2}-\mathbf{U}$.

\begin{tabular}{ccccccc}
\hline \hline & U-U & A & B & C & D & $T=300 \mathrm{~K}$ \\
\hline position / nm & 251.8 & 260.0 & 265.0 & 252.3 & 255.1 & 263.6 \\
$f$ & 0.21 & 0.15 & 0.15 & 0.10 & 0.17 & 0.15 \\
position / nm & 207.5 & 212.0 & 211.2 & 217.1 & 218.1 & 211.4 \\
$f$ & 0.27 & 0.33 & 0.29 & 0.29 & 0.29 & 0.30 \\
\hline \hline
\end{tabular}


Figure 1: $\mathbf{U}, \mathbf{U}-\mathbf{U}$, four stable conformers $(\mathbf{A}, \mathbf{B}, \mathbf{C}$, and $\mathbf{D})$ and two transition state structures of $\mathbf{U}-\mathrm{Ag}_{2}-\mathbf{U}$. Displacement vectors corresponding to the imaginary frequencies of the transition state structures, TS_AB (64.36i) and TS_AC (64.87i), are shown in blue lines. 
Figure 2: Calculated UV absorption spectra of (a) $\mathbf{U}-\mathbf{U}$ and four conformers of $\mathbf{U}-\mathrm{Ag}_{2}-\mathbf{U}$, and (b) $\mathbf{B}$ only and the spectrum of all the conformers in thermal equilibrium at $T=300 \mathrm{~K}$. The calculated spectra at various temperatures overlap with each other in this case. The lines are plotted assuming a Gaussian function with half width at half maximum (HWHM) of $0.2 \mathrm{eV}$.
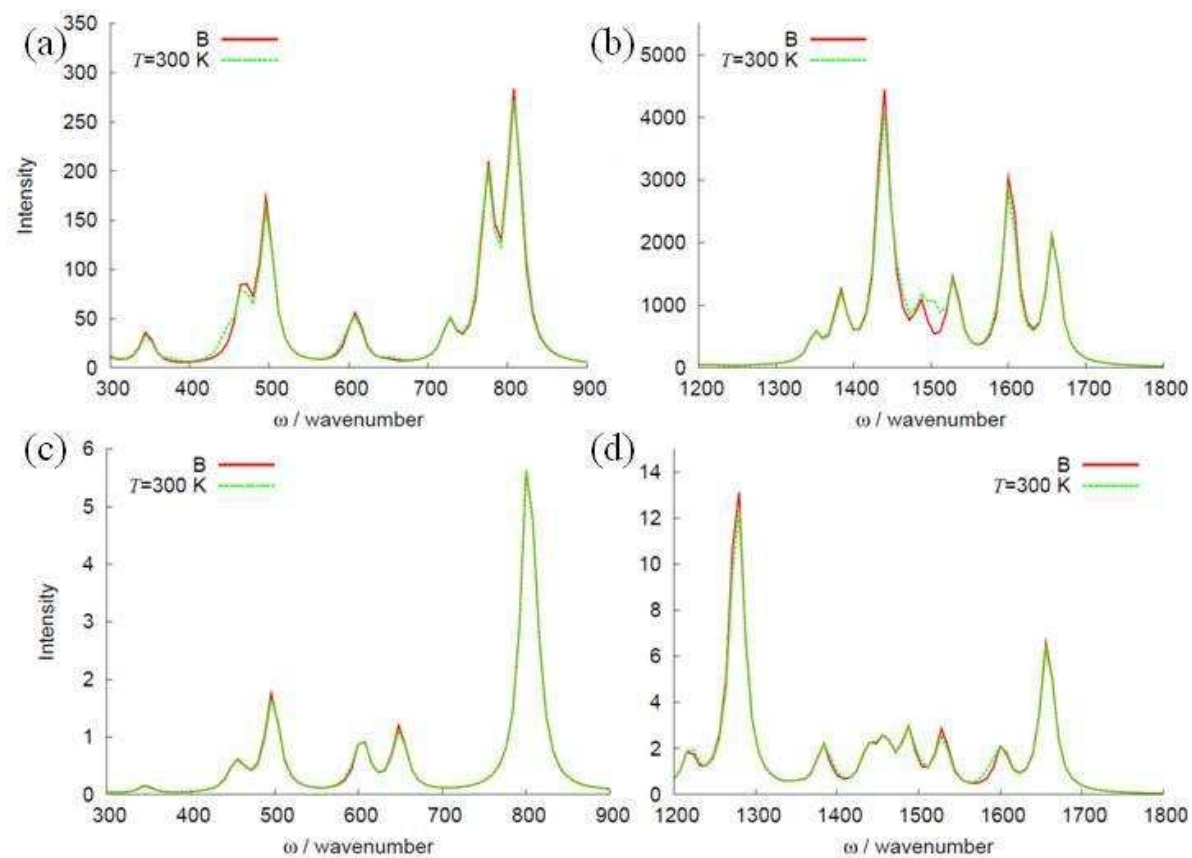

(d)

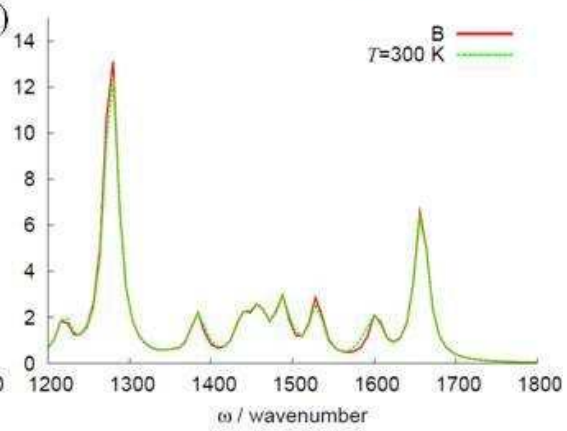

Figure 3: IR spectra (a, b) and Raman spectra (c, d) calculated for $\mathbf{B}$ only and those of all the conformers in thermal equilibrium at $T=300 \mathrm{~K}$. All spectra are plotted assuming a Gaussian function with $\mathrm{HWHM}$ of $10 \mathrm{~cm}^{-1}$. 
Graphical Abstract 1: This figure is for "Graphical Abstract": In the presence of $\mathrm{Ag}^{\mathrm{I}}$ ions, a mismatch DNA nuclobase pair, $\mathbf{U}-\mathbf{U}$, is expected to form four conformers of $\mathbf{U}-\mathrm{Ag}_{2}-\mathbf{U}$ each with two metal-nucleobase bridges. 